\title{
Priesterweihen Kölner Kleriker an der Kurie im 15. und 16. Jahrhundert.
}

Von

\author{
Dr. Ludwig Schmitz.
}

Im römischen Staatsarehive befinden sich neben einer Anzahl anderer Akten, die der apostolischen Kammer entstammen und aus denen in dieser Zeitschrift Heft 56, S. $144 \mathrm{ff}$. und 61, S. $129 \mathrm{ff}$. von $K$. Ha y n eine Reihe von Auszingen mitgetheilt worden sind, noch eine Serie Registerbände, die für die Provinzialgeschichte eine nicht weniger interessante Ausbente gewähren. Es sind die sog. Libri formatarun, deren für die Zeit von 1425-1524 sich 14 Bände erhalten haben. Ueber die änssere Einrichtung dieser Registerbände und ihren Inhalt habe ich in der "Römischen Quartalschrift für christl. Alterthumskunde und für Kirchengeschichte, 8. Jahrgang, 1894, S. $451-472$ ansfïhrlich gelıandelt. Indem ich in Uebrigen auf diesen Aufsat\% verweise, beschränke ich mich an dieser Stelle darauf, nur das Nothwendigste über diese Registerserie zu sagen, soweit es das Verständniss der unten folgenden Auszüge erheischt.

Der Name "Libri formatartu" riihrt daler, dass diese Kegister hauptsächlich die von den Kanonisten als "litterae formatae" bezeichneten Bescheinignngen uiber ertheilte Weiben - in unserem Falle also die an der Kurie ertheilten Ordinationen - enthalten. Daneben umfassen die Register noch eine andere Gruppe von Urkunden, die indess mit den erwähnten im engsten Zusammenhange stehen, nämlich die litterae dimissioriales oder dimissoriae. Dies sind, wie der Name schon besagt, Dispensbriefe: sie absolriren von der Beobachtung der einen oder anderen für die Rechtmässigkeit der Weihen sonst geltenden kanonischen Vorschrift; meistens 
geben sie dem Empfänger die Befngniss, sich von einem beliebigen Bischofe weihen zn lassen; andere erlauben den Empfang der Weihen an aussergewöhnlichen Tagen oder in schnellerer als der sonst gebräuchlichen Aufeinanderfolge. Einen grossen Raum endlich nimmt anch die Registrirung der allyemeinen Ordinationen ein. d. h. der nach den kirchlichen Verordnungen an den Samstagen der vier Quatember, dem Samstag Sitientes (vor Passionssomntagi) und dem Charsamstage regelmässig stattfindenden Weihen. Der Feier dieser "Ordines generales" wohnte jedesmal als Vertreter der Kammer, zu deren Ressort alle mit der Ertheilung von Weilıen zusammenhängenden Angelegenheiten gehörten, ein Notar bei. der dann die Registrirung der Weihen in urkundlicher Form vornahm.

Wohl aus allen Diözesen finden wir in diesen Registern zahlreiche Neopresbiter verzeichnet. So lernen wir aus ihnen auch ungefaihr 300 Angehörige der Kölner Diözese ${ }^{1}$ ), die an der päpstlichen Furie die verschiedenen Stufen der Weihe mehr oder weniger vollständig empfingen, für den Zeitraum ron 100 Jahren kemnen. Diese Zahl ist angesichts der grossen Ausdehnung der Erzdiözese gewiss nicht gross zu nemnen. Aber wir müssen dabei eben herücksichtigen, dass die Register einerseits nicht vollständig. erhalten sind, audererseits nicht alle Namen der Geweihten verzeichnen $\%$. Freilich ist es unmöglich, die nicht registrirten Weihen zahlenmässig zu schätzen, besonders weil seit 1501 die Beurkundung der "Ordines generales" wegfällt ${ }^{8}$ ).

Die unten mitgetheilten kurzen Ausziige sind der Uebersichtlichkeit und Raumersparniss wegen nach einem Schema bearbeitet. Auf den Namen des Geweihten folgt zunächst die Angabe der Weihe - ob Tonsur, niedere Weihen, Subdiakonat u. s. w. sodam das Datum, wann sie stattfand. Der Ort der Weihe ist

1) Es sei darauf hingewiesen, dass nach dem Sprachgebrauch der Kure unter "clericus Coloniensis dioc." jedesmal ein durch Geburt dem Gebiet der Kölner Diözese angehöriger Kleriker zu verstehen ist, mag er sich aufhalten, wo er will; clericus Coloniensis ist dagegen ein aus der Stadt Köln gevürtiger Kleriker. In äbnlicher Weise bedeutet "ecclessa sancti Martini Coloniensis" die Kirche St Martin in der Stadt Köln; ecclesia Coloniensis, ohne weiteren Zusatz, ist die Hauptkirche Kölns, der Dom.

2) Vergl. Römische Quartalschr. a. a. O. S. 457 n. $470 \mathrm{ff}$.

3) a. a. O. S. 461. 
natürlich abhängig von dem jeweiligen Aufenthaltsorte der Kurie; und zwar sind die Weihen unter Nr. 1-8 in Rom, Nr. 9-15 in Florenz, Nr. 16-26 in Bologna, Nr. 27--29 in Ferrara, Nr. 30 bis 35 wiedermm in Florenz, Nr. 36 in Siena und von Nr. 37 an alle in Rom ertheilt worden. Für alle Weihen ist uns anch der Name des ordinirenden Bischof's wie auch die Kirche, in der sie vor sich gingen, bekannt; beide jedesmal besonders anzuführen wäre aber $九 u$ weitläufig gewesen und hätte auch kaum Zweck gehabt, da ihre Mittheilung schwerlich für die Biographie u. s. w. des Geweihten von Interesse sein dürfte. - Die Fälle, in denen unsere Notizen aus einer eingetragenen Littera formata entnommen sind, werden durch die eingeklammerten Buchstaben (LF) mit dem Datum der Ansstellung dieser Bescheinigung gekennzeichnet ${ }^{1}$ ). Dem da eine derartige littera formata wallrscheinlich in der Regel nur auf besonderes Verlangen des Geweihten, \%. B. bei seinem Weggang von der Kurie, ausgestellt wurde ${ }^{2}$, so kann das betr. Datum für die Biographie von Bedeutung sein. Fehlt die Klammer (LF), so handelt es sich um Ausziige aus den nur zu Kämmereizwecken erfolgten Eintragungen der Ordines generales.

In den Anmerkungen sind wenigstens eine Anzahl von Persönlichkeiten identifizirt worden; jedoch habe ich mich hicrbei hauptsächlich auf Kenssen, Matrikel der Universität Köln citirt nach Rektorat und Nummer), auf die bereits erwähnten Auszïge ans den Annatenregistern ron $\mathrm{Hay}$ n (citirt: $\mathrm{H}$ a y n mit Nummer des betr. Regests) und die ersten 60 Hefte dieser Zeitschrift beschränkt. Die von Toepke herdusgegebene Matrikel der Universität Heidelberg (3 Bde., Heidelberg 1884 ff.) und das Repertorium Germanicum, Bd. I, Berlin 1897, die ebenfalls verglichen wurden, waren weniger ergiebig. Alle sonstigen etwa in Betracht kommenden Werke, besonders die lokalgeschichtlichen, zu diesem Zwecke heranzuziehen, war aher nicht möglich. Eine ansgedehntere Feststellung der einzelnen Persönlichkeiten muss der Lokalgeschichtsforschung überlassen werden, und das um so mehr, als bekanntlich gerade die Italiener in der falsehen Schreibung und Entstellung deutscher Namen vielfach fast unglaubliches geleistet haben.

1) Dieses Datum ist nicht besonders bemerkt, wenn es - wie meist - identisch ist mit dem der zulotzt ertheilten Weihe.

2) a. a. O. S. 471 . 
acol. = acolitus

b. = beate bezw. beati

can. = canonicus

cap. $=$ capellanus

cler. $=$ clericus

Col. dioc. $=$ Coloniensis diocesis

diac. $=$ diaconus

eccl. $=$ ecclesia
A blïrzungen.

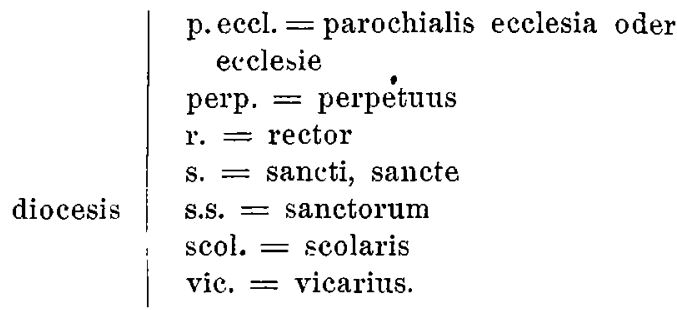

1. Theodericus Kempen, r. altaris b. Catharine et Nicholai in eccl. de Brakelen Col. dioc., acol. - Subdiak.: 9. Nor.; Diak.: 10. Nov.; Presbit.: 11. Nov. 1425.

2. Henricus Godde de Attendorn, r. p. ecel. in Menden Col. dioc. - Subdiak.: 21. Dez. 1426.

Keussen 84, 28; Rep. I, no. 389, 587, 859: Kanonikus an St. Andreas in Köln, Vikar in Mainz u. Strassburg; Ha y n 174, doch ist Menden $\mathrm{Kr}$. Iserlohn gemeint.

3. Johannes Deghen, r. p. eccl. in Mederic, Col. dioc. - Subdiak.: 23. Febr. 1427.

Keussen 175, 19; Hayn 104, 107, 419, 622.

4. Mathias Mathie Col. dioc. - Tonsur u. Akol.: 11. März 1430.

5. Johannes Itowe Col. dioc. - ebenso.

6. Tielmannus de Droilshagen, decanus et can. eccl. s. Martini in Muntstermeyfelt Trererensis dioc., subdiac. Col. dioc. Diak.: 11. März 1430.

Keussen 109, 10; Rep. I, 1147; Hay n 6, 46, 70, 83.

7. Sibertus Siberti de Waectendonch cler. Col. dioc. Akol.: 10. Juni 1430.

Keussen 170,54; Hayn 39, 170; Rep. I, 1340: r. p. eccl. in Sonchelen = Süchteln.

8. Henricus de Nuenkerken diac. Col. dioc. - Presb.: desgl. Keussen 73,1 oder 125,3 ?

9. Henricus de Horne Col. dioc. - Tonsur u. Akol.: 18. Dez. 1434.

10. Johannes Sprovemann cler. Col. - Subdiak.: 15. Mai 1435.

11. Johannes de Werda, can. eccl. s. Andree Col. - Diak.: desgl.

Joh. Cabele de Werda 1431 Kanonilus in Xanten, nach Annatenregister $I X, f .43 v$.

12. Johannes Upgestendonck Col. dioc. - Subdiak.: 24. Sept. 1435.

Hayn 567 ? 
Priesterweihen Kölner Kleriker an der Kurie im 15. u. 16. Jahrh. 95

13. Johannes Lessenich, cap. Gol. - Diak.: desgl.; Presb.: 17. Dez. 1435.

Hay $n$ 447?

14. Hermannus Becheler Coloniensis. - Akol.: 17. Dez. 1435.

15. Johannes Logner Col. dioc. - Subdiak.: desgl.

16. Johannes Paderbarn Col. dioc. - Tonsur u. Akol.: 2. Juni 1436 .

Stammt wohl aus Soest, vgl. Keussen II, 44.

17. Henricus Sthope de Unna Col. dioc. - Tonsur: 16. März 1437.

18. Johannes Oolhase Col. dioc. - Tonsur u. Akol.: desgl.

19. Fredericus Lair acol. Col. erhält Vollmacht, sich von jedem beliebigen kath. Bischofe zum Priester weihen zu lassen. Bologna, 3. Juli 1437.

20. Theodericus Sniwint de Heinsbergh, x. p. eccl. in Mechelen, diac. - Presb.: 25. Juli 1437.

Keussen 149,27; Hayn 397, 495.

21. Gerardus de Bellichoven Col. dioc. - Tonsur: 21. Sept. 1437.

22. Hermannus Ritter Col. dioc. - Tonsur und die niederen Weihen: desgl.

23. Goswinus de Bresteden Col. dioc. - Niedere Weihen und Subdiak. : desgl.

24. Henricus Henrici de Brilon Col. dioc. - Subdiak. : desgl. Kieussen 171,26 .

25. Wilhelmus Mirebet Col. dioc. - Tonsur u. Akol.: 21. Dez. 1437 .

1442 in Hejdelberg, T o epke I, 236. (?)

26. Johannes Millequinque (?) Col. dioc. - desgl.

27. Bernardus Wocger de Seden, plebanus in Sulen Col. dioc. - Subdiak.: 7. Juni 1438.

28. Wilhelmus de Glincohlt Col. dioc. - Tonsur: 20. Sept. 1437.

29. Hermannus Guther Col. dioc. - Tonsur: 20. Dez. 1438.

30. Henricus van der Heyden Col. dioc. - Diak.: desgl. can. Col. Presbit.: 21. März 1439.

Später Abt von Kloster Camp, Annalen 20, S. 369, 377, 378.

31. Bruno Spruten scol. Col. dioc. - Tonsur u. Akol.: 30. Mai 1439 .

1445 Prokuratur des Herzog Adolf von Cleve-Mark an der Kurie, Hansen, Rheinland und Westfalen I, S. 164. 
32. Mathias Len de Sonsbeeck Col. dioc. - Tonsur u. niedere Weihen: 17. Dez. 1440.

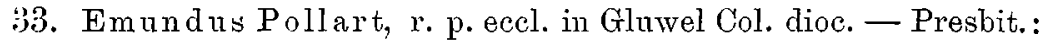
10. April $14+1$.

Hayn 135; nach Zeitschr. des Berg. Geschichtsvereins. IV, S. 265 c. 1447 Kanonikus von S. Maria in Kapitol in Köln und Pfarrer in Keyenberg:

34. Johannes de Berghausen (auch Joh. de Colonia alias de Berguehusen, Joh. de Bergerhusen), r. p. eccl. in Versen Col. dioc. - Subdiak.: 14. Juni 1441; Diak.: 23. Sept. 1441; Presbit.: 23. Dez. 1441 .

Keussen 138, 77; Norrenberg, Dek. Gladbach S. 131.

3อ̃. Nicolaus Le ekwick cler. Col. dioc. - Akol.: 24. Febr. 1442; can. s. Victoris Xantensis Subdiak.: 31. März 1442.

36. Bertoldus Nes cler. Col. dioc. - Akol.: 6. April 1443; r. p. eccl. cle Doruspick Traiectensis dioc. Subdiak.: 20. April 1443; Diak.: 15. Juni 1443 .

37. Tudnvicus de Berekoven Col. - Akol.: 19. Dez. 1444.

36. Tollannes Boynch can. eccl. b. Marie Reyssensis Col. dioc. - Subdiak.: 18. Dez. 1445́; Diak.: 12. 1rüz 1446; Presbit.: 2. April 1446 .

39. Johannes Sluper vic. s. Gereonis Col. - Niedere Weihen n. Subdiak.: 12. Mïizz 1446. Hayn $392,437,439$.

40. Henricus de Aenholt vic. s. Caterine in eccl. s. Marie de Binen Col. dioc. - Snbdiak.: 12. Mrirz 1446; Presbit.: 11. Juni 1446.

41. Willermus Willermy de Regno Col. dioc. - Diak.: 8. April 1447 .

42. Henricus Henrici scol. Col. - Tonstur: 33. Juni 1447: Akol. u. niedere Weihen: 28. Febr. 1450.

43. Johannes Rutze Col. dioc. - Tonsur: 23. Dez. 1447; Akol. u. niedere Weihen: 22. Dez. 1453.

44. Henricus Vandenoever Col. dioc. - Tonsur n. niedere Weihen: 23. Dez. 1447.

45. Johannes Weythase Col. dioc. - Tonsur: 17. Febr. 1448. Keusen 93, 21; Hayn 398, 620.

46. Henricus Rotgeri Col. dioc. - desg].

47. Henricus Loterij Col. dioc. -- Niedere Weihen: desgl.

48. Johannes Urbighen Col. dioc. - Tonsur: 18. Mai 1448 
Priesterweihen Kölner Kleriker an der Kurie im 15. u. 16. Jahrh. 97

49. Johannes Everardi scol. Col. dioc. - Tonsur: 21. Sept. 1448. Ke ussen 231,8 . (?)

50. Johannes Herinck Col. dioc. - Die niederen Weihen: desgl.

51. Gerardus de Ligno scol. Col. dioc. - Tonsur: 10. Dez. 1448. (L. F.)

52. Gerardus Sughendonch Col. dioc. - Tonsur: 21. Dez. 1448.

53. Johannes Hardor cler. Col. - Niedere Weihen: desgl.

04. Winandus Betzeler alias Agripinus, $x$. p. eccl. de Wirssen Col. dioc. - Subdiak.: 8. März 1449; Presbit.: 12. April 1449.

Ђō. Henricus de Wil Col. dioc. - Subdiak.: 20. Dez. 1449.

56. Theodericus Stoch Col. dioc. - Tonsur und niedere Weihen: 28. Febr. 14500.

57. Gerardus Harnon Col. dioc. - desgl.

58. Guillex m u Holland Col. dinc. - Nieclere Weihen: desgl.

59. Johanes Theoderici Col. dioc. - desgl.

60. Joh annes $\mathrm{N}$ a pe Col. dioc. - Presbit.: 4. Apri. 1450.

Keussen 217, 14; Hayn 451, 479; Toepke I, S. 205.

61. Guillelmus de Stan Col. dioc. - desgl.

62. Hermanu us de $\mathrm{H}$ a m m o ne alias Yde Col. dioc. - Niedere Weihen: 19. Dez. 1450 .

Ḱeussen 292, ว̆l.

63. Tilman $n$ u $M$ or is de W a I d orp cap. ad altare s. Rinoldi Col. - Subdiak.: desgl.

b4. Petrus Scurman Col. dioc. - Niedere Weihen: 20. März 1451. - x. par. eccl. in Rodenkirchen Subdiak.: 10. April 1451; Diak.: 24. April; Presbit.: 19. Juni 1451.

bŏ. Aruoldus Darte Col. dioc. - Tonsur: 10. Aprıl $145 \check{1}$.

b6. Henricus Brunonis canl. et scolasticus eccl. Werdensis Col. dioc. - Presbit.: 24. April 14001.

Hay $240,486,610$.

67. Sanderus Scabruch Col. dioc. - Tonsur u. niedere Weihen: 18. Sept. $140 ̄ 1$.

68. Gobelinus Flessen de Buren can. eccl. s. Victoris Xanctensis Col. dioc. - Subdiak.: 18. Sept. 1451; Diak.: 18. Dez. 145̃1; Presbit.: 4. März 1452.

Hayn $\mathbf{5 3 5}, 539$.

69. Walnerus Stelxen (oder Sterlen de Borchen) can. eccl. Col. - Subdiak.: 18. Sept. 1451; Presbit.: 18. Dez. $145 \check{1}$.

70. Gerardus de Capella, r. p. eccl. in Much Col. dioc. - Diak.: 18. Sept. 14ō1: Presbit.: 18. Dez. $140 ̄ 1$. 
71. Henricus Wissel Col. dioc. - Tonsur: 18. Dez. 1451.

72. Hermannus de Domstorpe can. eccl. s. Victoris Xanctensis Col. dioc. - Niedere Weihen: 18. Dez. 1451; Diak.: 25. März 1452; Presbit: 8. April 1452.

73. Johamnes Moer can. eccl. s. Victoris Xanctensis Col. dioc. - Niedere Weihen n. Subdiak.: 18. Dez. 1451; Presbit.: 8. April 1452.

74. Simon Moer Col. dioc. - Niedere Weihen: 18. Dez. 1451. Hayn 539.

75́. Petrus Squelpe can. Xanctensis Col. dioc. - Subdiak.: 25. März 1452.

Ob Hayn $2=$ Petrus Schalpipe? Vergl. über diesen Rep. I, no.

76. Guillelmus de Brede utriusque iuris doctor, prepositns s. Cuniberti Col. - Diak.: 25. März 1452.

Hayn 531 u. 532. - Rep. I, 2487.

77. Walterus $\mathrm{Krag}$ r. p. eccl. sive personatus in Wreghen Col. dioc. - Diak.: 25. März 1452; Presbit.: 8. April 1452. Hayn 397.

78. Johannes Lenepe scol. Col. dioc. - Tonsur u. niedere Weihen: 8. April 1452; r. p. eccl.. in Stommel Subdiak.: 21. Dez. 1454; Diak.: 10. März 1455.

Keussen 289,95 ?

79. Alfonsus Benhughen Col. dioc. - Niedere Weihen: 8. April 1452.

80. Johannes Delo Col. dioc. - Tonsur: 3. Juni 1452.

81. Magister Henricus Huseman r. p. ecel. in Bostorp Col. dioc. - Subdiak.: 23. Sept. 1452.

Hayn 419, 412; Keussen 224, 12.

82. Arnoldus Meheter Col. dioc. - Niedere Weihen: 23. Dez. 1452.

$\mathrm{H}$ a y $\mathrm{n} 457$.

83. Willelmus de Sarbrucken Col, dioc. - Tonsur: 24. Febr. 1453; Akol. u. niedere Weihen : 17. März 1453; Subdiak.: 22. Dez. 1453; capellanus Kryneczbach nuneupatus Diak.: 16. März 1454.

84. Nicolans Moer vic. ad altare trium regum Bunense Col. dioc. - Niedere Weihen: 24. Febr. 1453.

85̄. Ludovicus Gerwini Col. dioc. - Tonsur: 17. März 14503.

86. Goswinus de Vecten Col. dioc. - Tonsur: 26. Mai 1453. 
Priesterweihen Kölner Kleriker an der Kurie im 15 u. 16. Jahrh. 99

87. Jo hannes $\mathrm{N} u l$ Col. dioc. - desgl.

88. Johannes Ewoch Col. dioc. - Tonsur u. niedere Weihen 26. Mai 1453.

Hay $n$ 468, 615 .

89. Ghisbertus de Geer Col. dioc. - Niedere Weihen: 26. Mai 1453.

90. Mathias Nickelen de Euskirchen Col. dioc. - Subdiak.: 26. Mai 1453; cap. ad altare b. martirum Philippi et Jacobi situm in eccl. ss. Crisanti et Darie Monasteriensis Eyfflie. Presbit.: 6. April 1454.

Ob Hayn 463?

91. Johannes Mercatoris de Seggeroid Col. dioc. Niedere Weihen: 22. Sept. 1453.

92. Hermanu $\mathrm{s} \mathrm{Kram}$ er Col. dioc. - Tonsur: 22. Dez. 1453.

93. Vinricus Krul de Wushiem Col. dioc. - Tonsur u. niedere Weihen: 22. Dez. 1453.

Hayn 379. (?)

94. Rutherus (?) Berkys Col. dioc. - Niedere Weihen: 22. Dez. 14533.

95. Joh ann es d e $\mathrm{K}$ e mpen can. b. Marie Ressensis Col. dioc. - Subdiak.: 22. Dez. 1453.

96. Radulphus Winckelhusen scol. Col. dioc. - Tonsur: 6. April 1454.

97. Johannes Huysraet scol. Col. dioc. - Tonsur: 15. Juni 1454 .

98. Petrus Cornelii de Freris r. p. eccl. de Versen Col. dioc - Niedere Weihen: 15. Dez. 1454; Subdiak.: 21. Dez. 1454; Diak.: 10. März 1455; Presbit.: 5. April 1455. Hayn 601; Norrenberg a. a. O. S. 131.

99. Jacobus Wissechel alias Monick cler. Col. dioc. Niedere Weihen: 21. Dez. 1454.

100. Matheus Lobe perpetuus vic. ad altare b. Marie virg. in monasterio s. Cornelii Indensis O. S. B. Col. dioc. - Subdiak.: 21. Dez. 1454; Diak.: 10. März 1455; Presbit.: 22. März $145 \tilde{o}$.

101. Nicolaus Cremer de Bercha cler. Col. - Niedere Weihen: 27. Febr. 1455; Subdiak.: 10. März 1455; Diak.: 22. März 1455 ; Presbit.: 5. April 1455. 
102. Thomas de Berghen Col. dioc. - Niedere Weihen: 10. März 1455 .

Ob Keussen 209, 47?

103. Christianus Thysen scol. Col. dioc. - Tonsur: 22. Sept. 1464.

104. Theodericus Kystemeker de Xantis, cler. Col. dioc. Niedere Weihen: 22. Sept. 1464.

Keusien 235, 11.

105. Stephanus Greverode cler. Col. dioc. - desgl. Keussen $304,48$.

106. Arnoldus Dernen de Erpel, pastor in Winteren Col. dioc. - Niedere Weihen: 22. Sept. 1464; Diak.: 22. Dez. 1464; r. p. eccl. in Wuthicheren (?) Presbit.: 30. März 1465.

107. Guillelmus Nolden subdiac. Col. disc. - Diak. : 22. Sept. 1464.

108. Henricus D a Iman cler. Col. dioc., sanct. ${ }^{\mathrm{mi}}$ domini nostri pape cubicularius secretus. - Niedere Weihen: 12. März 1士66. (L. E.)

Haỹn 442, 448, 403, 488, 489, 490.

109. Petrus Vernudeken cler. Col. dinc. - Niedere Weihen: 22. Dez. 1464.

110. Albertus Cruse cap. capellanie in Geresheim Col. dioc. - Presbit.: 22. Dez. 1464.

111. Henricus de Ophus sen cler. Col. dioc. - Niedere Weihen u. Subdiak.: 9. März 1465; r. p. eccl. in Rinderen Diak.: 30. Mä̀z 1465 ; Presbit. : 13. April 1465. (L. F. 4 Mai 1471.) Hayn 407 [Pfarre Rindern bei Cleve!] 532; Keussen 297, 113.

112. Theodericus Undereykyn scol. Col. dioc. - Tonsur: 13. April 1465 .

Hayn 528 .

113. Johannes de $\mathrm{Pap}$ is scol. Col dioc. - Tonsur: 8. Juni 1465.

Hayn 503.

114. Ludovicus Crewin r. p. eccl. in Burgresthemc. (?) Col. dioc. - Subdiak.: 8. Juni 146 כ.

115. Win andus $R \circ g \mathrm{~g}$ em an cler. Col., perpetuus vic. in eccl. Lubicensi. -- Subdiak. : 21. Dez. 1465; Diak. : 1. März 1466; vic. Bremensis et Lubicensis ecclesiarum Presbit: 21. März 1466. 
Priesterweihen Kölner Kleriker an der Kurie im 15. u. 16. Jahrh. 101

116. Bartholomeus Goir scol. Col. dioc. - Tonsur: 1. März 1466 [L. F.].

Hayn 518.

117. L im bertus Waullerde Syborch, r. p. ecel. s. Georgii in Nyderpleyse - 4 niedere Weihen u. Subdiak. : 5 . April 1466.

118. Mathias Sartoris scol. Col. - Tonsur u. niedere Weihen: 31. Mai 1466.

119. Joh a n nes Ja charez scol. Col. dioc. - Tonsur: 20. Sept. 1466 .

120. A l bertus $\mathrm{Sch}$ om ken scol. Col. dioc. - Tonsur u. niedere Weihen: 20. Dez. 1466.

121. Johames Pistoris scol. Col. dioc. - Tonsur: 20. Dez. 1466.

122. Henricus Tossut cler. Col. dioc. - Niedere Weihen: 20. Dez. 1466.

123. Johannes Lens can. eccl. s. Andree Col. - Subdiak.: 28. Mä̀z 1467.

124. Johannes Ruscher de Xanctis scol. Col. dioc. - Tonsur: 23. Mai 1467. [L. F.]

125. Johannes Westerhoult scol. Col. dioc. - Tonsur: 19. Sept. 1467.

126. religiosus frater Jordanus de Uterwil domus s. Francisci de Valle Josephat Col. dioc. ord. minorum de observ. - Tonsur u. niedere Weihen: 7. Febr.; Subdiak.: 9. Febr.; Diak.: 10. Febr.; Presbit.: 14. Febr. 1468. [L. F.]

127. Mathias Kalthoff cler. Col., r. p. eccl. in Steldorp, acolitus. - Subdiak.: 12. Febr.; Diak.: 19. Febr.; Presbit.: 22. Febr. 1469. [L. F.]

128. Guillelmus Bolth (Boult) scol. Col. dioc. - Tonsur: 25. Febr. 1469. [L, F.]

129. Johannes Derne de Unna Col. dioc. - Tonsur: 25́. Febr. 1409 .

Ke ussen 249,18 .

130. Symon de Wientren Col. dioc. - Niedere Weihen: 23. Dez. 1469 .

Keusisen 257, 42.

131. Bruno Cluyt de Werda cler. Col. dioc., r. p. eccl. in Wijlre s. Gereonis. - Niedere Weihen: 19. Dez. 1470; Subdiak.: 
22. Dez. 1470; Diak.: 9. März 1470; Presbit.: 12. März 1470. [L. F. 13. März 1470.]

1465 Sept. 3 . in Rom nachweisbar als Familiar des Kardinals von Spoleto und Kanonikus in Münstermaifeld. $1467 \mathrm{Febr}$. 23. bezahlt er persönlich die Annate. Römisches Staatsarchiv Div. Pauli 1464-1465, fol. $162 \mathrm{v}$.

132. Mathias Marpotze, r. p. eccl. in Graenkyndorp (ob Stal(n)kyndorp = Selgersdorf ?) Col. dioc., acol. - Subdiak.: 1. Mai; Diak.: 3. Mai; Presbit.: 5. Mai 1471. [L. F.]

133. Johannes van der Lynden scol. Col. dioc. - Tonsur: 16. Juni 1470. [L. F.]

134. Johannes Lyndeman scol. Col. dioc. - desgl.

135. Hermannus Tepel scol. Col. dioc. - desgl.

136. Johannes Zeris Col. dioc. - Tonsur: 22. Sept. 1470.

137. Johannes Buchel de sancto Vito cler. Col. dioc., r, eccl. b. Marie in Novavilla alias in sancto Vito Leod. dioc. Niedere Weihen: 8. Mai; Subdiak.: 12. Mai; Diak.: 19. Mai; Presbit.: 26. Mai 1471. [L. F.]

138. Petrus Berchem scol. Col. dioc. - Tonsur: 21. Sept. 1471. 139. Johannes de L enep scol. Col. dioc. - desgl.

140. Gerardus Rint scol. Col. dioc. - Tonsur: 21. Dez. 1471.

141. Arnoldus Merspfer (Mersker, Melxter) Col. dioc. - Subdiak.: 22. Febr. 1472; Diak.; 14. März 1472; r. p. eccl. in Vamel Presbit.: 28. März 1472. [L. F.]

142. Guerwinus Micken r. p. eccl. in Brische Col. dioc. - Subdiak.: 22. Febr. 1472; Diak.: 14. März 1472.

Hay n $369,372,382,487$.

143. Christian us Worst alias de Castro Col. dioc. - Diak.: 22. Febr. 1472. - Presbit.: 14. März 1472.

144. Johannes de Petra scol. Col. - Tonsur: 28. März 1472.

145. Johannes Xihorus scol. Col. dioc. - Tonsur: 19. Sept. 1472.

146. Winandus Dyeteren, r. p. eccl. in Vetten Col. dioc. Niedere Weihen: 7. Nov.; Subdiak.: 8. Nov.; Diak.: 3. Nov.; Plesbit.: 1ら̄. Nov. 1472. [L. F.]

147. Henricus Hecht, r. p. eccl. in Dattenvelt Col. dioc. Niedere Weihen: 24. Dez.; Subdiak.: 26. Dez.; Diak.: 27. Dez. 1472. [L. F.] — Erhält Litt. dimiss. mit Erlaubniss, sich ibberall zum Priester weihen zu lassen: 1. Juni 1473. 
Priesterweihen KöIner Kleriker an der Kurie im 15. u. 16. Jahrh. 103

148. Petrus Syfridi de Wyringia r. hospitalis oppidi Juliacensis Col. dioc., cler. - Niedere Weihen: 29. Januar; Subdiak.: 30. Januar; Diak.: 2 Febr.; Presbit.: 6. Febr. 1473. [I.F.]

149. Hermannus Mor scol. Col. dioc. - Tonsur: 13. März 1473.

150. Johannes Schorn scol. Col. dioc. - Tonsur: 12. Juni 1473.

151. Petrus Welker can. Col. - Presbit.: 12. Juni 1473. [L. F. 25. Juni 1473: gratis pro coquo $\mathrm{r}^{\mathrm{mi}}$ domini Camerarii.]

152. Adrianus Theoderici de Forest, in artib. magister, cap. ad altare s. Crucis in eccl. s. Andree Col., clericus. - Niedere Weihen: 15. Aug.; Subdiak.: 29. Aug.; Diak.: 5. Sept.; Presbit.: 8. Sept. 1473. [I. F. 15. Sept. 1473.]

153. Henricus Terwyck scol. Col. - Tonsur: 18. Sept. 1473.

154. Sanderus Eyckpass de Kempen, r. p. eccl. s.... in Superiori Bachem Col. dioc., cler. - Niedere-Weihen: 1. Febr.; Subdiak.: 2. Febr.; Diak.: 5. Febr.; Presbit.: 6. Febr. 1474. [L. F.]

15̆5. Petrus Altichart r. p. eccl. s. Christofori Col. - Subdiak.: 5. März; Diak.: 6. März; Presbit.: 13. März 1474. [L. F.]

15́6. Arnoldus de Kersten (oder Kesteren) vic. perp. ad altare s. Antonii in p. eccl. de Horsen Col. dioc. - Niedere Weihen: Subdiak. u. Diak.: 4. Juni; Presbit.: 11. Juni 1474. [L. F.]

10̆7. Johannes Muschen scol. Col. dioc. - Tonsur: 21. Aug. 1474. [L. F.]

158. Andreas Haerberti (Herbord) scol. Col. dioc. - Tonsur: 24. Sept. 1474; r. p. eccl. in Monhem. - Niedere Weihen u. Subdiak.: 5. Juni; Diak.: 9. Juni; Presbit.: 10. Juni 1487. [L. F.]

159. Petrus Hilpot scol. Col. dioc. - Tonsur: 17. Dez. 1474; r. p. eccl. in Vyrssen Col. dioc. - Niedere Weihen u. Subdiak.: 17. April; Diak.: 23. April; Presbit.: 24. April 1485. [L. F.]

Hayn 601; Norxenberga. a. O. S. 131.

160. Petrus Persinan scol. Col. dioc. - Tonsur: 17. Dez. 1474.

161. Johannes Pelzjohannis perp. vic. eccl. Suzatiensis Col. dioc. - Subdiak.: 18. Febr.; Diak.: 11. März; Presbit.: 25. Närz 1475. [L. F.]

162. Johannes Greve de Kempis r. p. eccl. in Reide Col. dioc. - Presbit.: 18. Febr. 1475.

Keussen 280, 3 ; Hayn 452 . 
163. Guilhelmus Giusg in cler. Col., perp. vic. in eccl. s. S'everini Col. - Niedere Weihen u. Subdiak.: 28. Sept.; Diak.: 30 Sept.; Presbit.: 1. Okt. 1475. [L. F.]

164. Henricus Bocholdie r. p. eccl. in Woelrsschem Col. dioc. - Niedere Weihen u. Subdiak.: 12. März; Diak.: 13. März; Presbit.: 21. März 1479. [L. F. 22 März 1479.]

165. Johannes Garschen scol. Col. dioc. - Tonsur: 22. Sept. 1481.

166. Albertus Brochte scol. Col. dioc. - Tonsur u. niedere Weilen: 22. Sept. 1481.

167. Hermannus Rebsy (Rewsi) scol. Col, - Tonsur: 22. Dez. 1481. [L. F.]

168. Johannes Himelkron cap. ad altare trium regum in p. eccl. in Duisseltorp Col. dioc., acol. - Subdiak.: 2. Febr.; Diak.: 10. Febr, 1482. [L. F.]

169. Gabriel de Bryersze cler. Col. dioc., Newerteich Pomezaniensis et Elbingk Warmiensis dioc. parochialium ecclesiarum rector. - Subdiak.: 17. Febr.; Diak.: 22. Febr.; Presibit.: 24. Febr. 1482. [L. F.]

170. Martinus Trink scol. Col. dioc. - Tonsur: 2. März 1482.

171. Ivo de Orsoy scol. Col. dioc. - Tonsur: 21. Sept. 1482.

172. Gobellinus Juliaci scol. Col. dioc. - Tonsur: 20. Okt. 1482. [L. F.]

173. Henricus Reetz scol. Col. dioc. - Tonsur: 21. Dez. 1482; niedere Weihen: 22. Dez. 1492.

174. Johannes Doleatoris scol. Col. dioc. - Tonsur: 21. Dez. 1482.

175. Henricus Horsel cler. et vic. in eccl. ss. Apostolorum Col. - Subdiak.: 8. Juni; Diak.: 11. Juni; Presbit.: 21. Juli 1483. [L. F.]

176. Johannes Boesgen can. eccl. collegiate ss. Crisanti et Darie Col. dioc. - Niedere Weihen: 13. März 1484.

177. Guillelmus Gerlazii scol. Col. dioc. - Tonsur: 3. April 1484 .

178. Henricus Wida Col. dioc. - Niedere Weihen: 26, Febr. 1485.

179. Petrus Tilimanni Col. - Tonsur: 24. Sept. 1485.

180. Hermannus Sforstenberch Col. - Tonsur: 17. Dez. 1485. [L. F.] 
Priesterweihen Kölner Kleriker an der Kurie im 15. u. 16. Jahrh. 10a

181. Petrus Michaelis cler. Col. dioc., r. p. eccl. s. Petri in Deytkirchen dicte dioc. - Subdiak.: 18. Dez.; Diak.: 21. Dez.; Presbit.: 27. Dez. 1485. [L. F. 30 Dez. 1485.]

182. Eustachius Segrado perp. vic. ad altare s. Cormelii in monasterio s. Cornelij Indensi Col. dioc., cler. - Niedere Weihen: 28. Januar; Subdiak.: 2. Febr.; Diak.: 3. Febr. 1486. [L. F. 6. Febr. 1486.]

Stammt wohl aus Aachen, vergl. Annalen 57, S. 111, no. 563.

183. Antonius Stenus scol. Col. dioc. - Tonsur: 18. Febr. 1486. [L. F.]

184. Johannes $\mathrm{Schuman}$ perp. vic. ad altare s. Agathe in colleg. eccl. Bonnensi Col. dioc. - Niedere Weihen ц. Subdiak.: 21. März; Diak.: 26. März; Presbit.: 27. März 1486. [L. F.]

185. Henricus Keymerlinck cap. capelle b. Marie Ville Kersmich Col. dioc. - Niedere Weihen u. Subdiak.: 25. März; Diak.: 29. April; Presbit.: 1. Mai 1487. [L. F. 12. Mai 1487.]

186. Johannes Fortis scol. Col. dioc. - Tonsur und niedere Weihen: 31. März 1487.

187. Petrus Smedt, vic. altaris b. Marie virg. in eccl. s. Johannis Baptiste Col. - Subdiak. : 14. Olkt.; Diak.: 18. Okt.; Presbit.: 21. Oct. 1487. [L. F.]

188. Matheus de Roda perp. vic. ad altare b. Marie in colleg. eccl. s. Cuniberti Col. - Niedere Weihen: 5. April; Subdiak.: 7. April; Diak.: 8. April; Presbit.: 13. April 1488.

189. Wilhelmus de Wichen cler. Col., in artibus magister, perp. vic. ad altare s. Antonii in eccl. s. Mauritii Col. - Niedere Weihen: 20. April; Subdiak.: 23. April; Diak.: 27. April; Presbit.: 1. Mai 1488. [L. F. 2. Mai 1488.]

190. Theodericus Spijst, perp. beneficiatus ad altare b. Petri apost. in p. ecel. de Haren Col. dioc. - Subdiak. : 6. Juli; Diak.: 7. Juli; Presbit.: 13. Juli 1488. [L. F.]

191. Johannes Greyff perpetuus cap. sen vic. in Lubrich Col. dioc., artium baccalareus. - Subdiak.: 19. Okt.; Diak.: 26. Okt.; Presbit.: 28. Okt. 1488. [L. F.]

192. Gaufridus Iffardi r. p. eccl. s. Cuniberti Col. - Subdiak.: 19. Juli; Presbit.: 22. Juli 1489 [L. F.]

193. Johannes $\mathrm{K}$ oningeshoeven perp. vic. ad altare s. Crucis in p. eccl. in Lipelerer Col. dioc. - Subdiak.: 11. April; Diak.: 18. April; Presbit.: 20. April 1489. [L. F.] 
194. Thomas Rademecher r. p. eccl. in Esberch Col. dioc. Subdiak.: 7. Juni; Diak. : 9. Juni ; Presbit. : 11. Juni 1489. [L. F.]

195. Hermannus $\mathrm{K}$ a el cler. Col. dioc., r. p. eccl. s. Martini in Hugert. - Niedere Weihen u. Subdiak.: 22. Juli; Diak.: 25. Juli; Presbit.: 30. Juli 1489. [L. F.]

196. Johannes $\mathrm{Helwingen}$ cap. ad altare b. Marie virg. in p. eccl. in Guterswyck Col. dioc. - Niedere Weihen: 25. Okt.; Subdiak.: 28 Okt.; Diak.: 1. Nov.; Presbit.: 8. Nov. 1489 [L. F.]

197. Goeswin us Noeck cler. Col. - Subdiak. und Diak.: 8 Nov.; Presbit.: 9. Nov. 1489. [L. F. 11. Januar 1490.]

198. Henricus Theoderici, acol. et can. colleg. eccl. ss. Crisanti et Darie in Monasterio Eiffalensi. - Subdiak.: 6. Dez.; Diak.: 7. Dez.; Presbit.: 8. Dez. 1489. [L. F.]

199. Petrus Vecodertroppen cler. Col. dioc., r. capelle s. Cecilie in Hubenredt. - Niedere Weihen: 21. Febr.; Subdiak.: 22. Febr.; Diak.: 24. Febr.; Presbit.: 28. Febr. 1490. [L. F.]

200. Andreas $\mathrm{Sw}^{\mathrm{a}}$ olis can. ecel. b. Marie in Capitolio CoI. Diak.: 4. April ; Presbit.: 7. April 1490. [L. F.]

201. Ma uritius Pernis cap. ad altare trium Regum in eccl. Col. - Tonsur u. niedere Weihen: 23. April; Subdiak.: 1. Mai; Diak.: 2. Mai ; Presbit.: 3. Mai 1490. [L. F.]

202. Hermannus Rorbach, r. p. eccl. ss. Petri et Panli Col. dioc. - Diak.: 5̃. Juni 1490.

203. Henricus Hevessenberg cap. in eccl. b. Marie Keiemburgensis. - Subdiak.: 18. Sept. 1490.

204. Henricus Buck de Osnaburg perp. vic. ad altare b. Marie in Hachemburg Col. dioc. - Diak.: 18. Sept.; Presbit.: 21. Sept. 1490. [L. F.]

205. Petrus Steynt de Blensze cler. Col., perp. cap. s. Cuniberti in Ulperiich prope Tulpetum dicte dioc. - Subdiak.: 13. Nov.; Diak.: 18. Nov.; Presbit.: 21. Nov. 1490. [I. F.]

206. Theodoricus Hattemarus scol. Col. dioc. - Tonsur: 18. Dez. 1490 .

207. Petrus Sten (Steyn) cler. Col. dioc. - Niedere Weihen: 18. Dez. 1490; cap. s. Cuniberti Subdiak.: 23. April; Diak.: 24. April; Presbit.: 26. April 1491. [L. F.] 
Priesterweihen Kölner Kleriker an der Kurie im 15. u. 16. Jahrh. $10 \tau$

208. Guerhardus Herzhuisen, beneficiatus provisus in patrimonio in Vesalia inferiori Col. dioc. - Niedere Weihen: 16. Januar; Subdiak.: 17. Januar; Diak.: 20. Januar; Presbit.: 21. Januar 1491 [L. F.]

209. Cornelius Nuyssen de Krutzenich in artibus mag., ric. seu cap. perpet. ad altare s. Johannis evang. in p. eccl. de Roden ante Silvam Col. dioc. - Subdiak.: 6. März; Diak.: 7. März; Presbit.: 12. März 1491. [L. F.]

210. Arnoldus Tulpeti acol, perp. vic. ad altare s. Crucis in p. eccl. s. Lisolfi Col. - Subdiak.: 12. März; Diak.: 13. März: Presbit.: 19. März 1491. [L. F.]

211. Johannes Lirgis de Nidegen, perp. cap. simplicis et ruralis capellanie s. Nicolai Steynbuchel Col. dioc. - Subdiak.: 2. April: Diak.: 24. April; Presbit.: 25. April 1491. [L. F.]

212. Wilhelmus Kremer cler. Col. dioc., perp. vic. ad altare s. Antonii in p. eccl. in Halderen Col. dioc. - Niedere Weihen: 28. Mai; Subdiak.: 12. Juni; Diak.: 19. Juni; Presbit.: 26. Juni 1491. [L. F.]

213. fr. Johannes de Ercklens Col. dioc. - Presbit.: 28. Mai 1491.

214. Mathias Haler perp. cap. in capella s. Agathe in Molenanch Col. dioc. - Niedere Weihen: 17. März; Subdiak.: 18. Mårz: Diak.: 21. März; Presbit.: 25̄. März 1492. [L. F.]

215. Johannes Kopp de Nussia cler. Col., r. p. eccl. in Phanert (?) — Niedere Weihen: 4. März; Subdiak.: 12. März; Diak.: 17. März; Presbit.: 18. März 1492. [L. F.]

216. Henricus Sondoch de Tulpeto, perp. vic. p. eccl. s. Petri de Tulpeto Col. dioc. - Subdiak.: 8. Sept.; Diak.: 9. Sept.; Presbit.: 14. Sept. 1492. [I. F.]

217. Johannes Schlossert, perp. cap. ad altare s. Marcelli Col. - Diak.: 19. Okt.; Presbit.: 21. Okt. 1492 [T. F.]

218. Vernerus Scolaris, perp. beneficiatus in eccl. Villenove ad altare b. Marie Col. dioc. - Tonsur und niedere Weihen: 22. Nov.; Subdiak.: 25. Nov.; Diak.: 30. Nov.; Presbit.: 2. Dez. 1492. [L. F.]

219. Petrus Bilgenbach, cler. Col. dioc., perp. vic. ad altare s. Catherine in Minbach dicte dioc. - Subdiak. :22. Nov.; Diak.: 25. Nov.; Presbit.: 30. Nov. 1492. [L. F.] 
220. Henricus Meyerspach, perp. cap. ad altare gloriosissime virg. Marie in conceptione in p. eccl. Adenare Col. dioc. Subdiak.: 30. Nov.; Diak.: 2. Dez.; Presbit.: 6. Dez. 1492. [L. F.]

221. Henricus Vinkel, perp. cap. ad altare b. Marie virg. extra muros Andernacenses. - Subdiak.: 30. Nov.; Diak.: 2. Dez.; Presbit.: 6. Dez. 1492. [L. F.]

222. Aluoldus de Vulff clel. Tomacensis dioc. ac perp. vic. capellanie ad altare b. Marie Magdalene in p. eccl. oppidi in Rechelinchusen Col. droc. - Niedere Weihen: 30. Nov.; Subcliak.: 2. Dez.; Diak.: 6. Dez.; Presbit.: 8 Dez. 1492. [T. F.]

223. Johannes Ponger scol. Col. dioc. - Tonsur: 2. März 1493.

224. Antonius Git, vic. ad altare s. Antonii in eccl. b. Brigide Col. -- Subdiak.: 23. März 1493.

225. Otto de Specll can. s. Cassii Bonnensis Col. dioc. - Subdıak.: 2. März; Diak.: 23 März; Presbit.: 6. April 1453.

226. Hadruanus Hissboutz Col. dioc. - Niedere Weihen: 6. April 1493 .

227. Elbertus Vrede cler. Col. dioc. - desgl.; r. p. eccl. s. Odolricu in Alpen Col. dioc. Subdiak.: 27. Febr.; Diak.: 19. Màrz; Presbit : 2. April 1496.

228. To ha an es de Pasqua scol. Col. dioc. - Tonsur: 1. Juni 1493 .

229. Ad am Chasbach scol. Col. dioc. - Tonsur: 14. Sept. 1493. [L. F.]

230. Armoldus Stap pert scol. Col. dioc. - Tonsur: 21. Dez. 1493 .

231. Patroclus Teveren scol. Col. dioc. - desgl. [L. F.]

232. Thomas Michaelis scol. Col. dioc. - desgl.

233. Johannes Tithrius cler. Col. dioc. - Niedere Weihen: 29. März 1494.

234. Johannes Haron scol. Col. dioc. - Tonsur: 24. Mai 1494.

235; Johannes Pruys cler. Col., acol., vic. in ecc. p. s. Martini minoris Col. ad altare s. Agnetis. - Subdiak.: 14. März; Diak.: 15̄. März; Presbit.: 25. März 1495.

236. Wilhelmus Langeraet, perp. vic. altaris s. Blasii supra portam in eccl. s. Michaelis Col. - Niedere Weihen u. Subriak.: 23. April; Diak.: 26. April; Presbit.: 3. Mai 1495. [L. F.] 
Priesterweihen Kölner Kleriker an der Kurie im 15. u. 16. Jahrh. 109

237. Johannes Ingeraud, perp. vic. ad altare b. Marie virg. in eccl. s. Michaelis Col. - ebenso.

238. Petrus Wide scol. Col. dioc. - Tonsur: 13. Juni 1495.

239. Johannes Piffer, cler. Col. dioc., l. perp. capellanie ad altare s. Carentii in p. eccl. Alsdorff, Col. dioc. - Niedere Weihen u. Subdiak.: 13. Sept.; Diak.: 14. Sept.; Presbit.: 18. Sept. 1495. [L. F.]

240. Georgius Stengel, scol. Col. dioc. - Tonsur: 27. Febr. 1496.

241. Henricus Geberti de Piuffelick Col. dioc. - Tonsur: 18. März 1496.

242. fr. Jacobus Coloniensis or. s. Brigide Jannensis. - Diak.: 18. März; Subdiak.: 2. April 1496.

243. Gerardus Scolten scol. Col. dioc. (Schnolt, Scholt). - Tonsur u. niedere Weihen: 2. April 1496; altarista ad altare S. Lucie in eccl. s. Antonii .... (unleserlich). - Subdiak.: 22. Mai; Diak. : 23. Mai : Presbit.: 24. Mai 1496. [I. F.]

244. Carolus de Eylle scol. Col. dioc. - Tonsur n. nedere Weihen: 2. April 1496.

245. Johannes Fluerenti $B$ raxatoris pelp. cal. altaris b. Marie virg. et s. Agathe in p. eccl. s. Lamberti in Emuntroide Col. dinc. - Subdiak.: 17. April; Dık.: 24. April; Presbit.: 25. April 1496. [L. F.]

246. Johannes Cronenburgh, perp. cap. capelle n. Gritii (ob Briccii?) in Udempret Col. dioc. - Subdiak.: 23. April; Diak.: 25. April; Presbit.: 1. Mai 1496. [L. F.]

247. Henricus Siberti vic. perp. an altare $s$. Gatherine in p. eccl. de Vanchem Col. dioc. -. Tonsur, niedere Welhen u. Subdiak.: 1. Mai; Diak. : 3. Mai; Presbit. : 5. Mai 1896. [L. F.]

248. Arnoldus de Wersthoren perp. altarista altaris b. Marie virg. in p. eccl. s. Nicolai in Asboch Col. dioc. - Sinbdiak.: 6. Mai; Diak.: 12. Mai ; Presbit.: 15. Mai 1496. [L. F.]

249. Gotfridus $\mathrm{K}$ orff, perp. cap. capelle s. Leonardi $m$ Hilwart Col. dioc. - Subdiak.: 22. Mai; Diak.: 23. Mai: Preslıt.: 24. Nai 1496. [L. F.]

250. Henricus Wrede junior seol. Col. dioc. - Tonsm und niedere Weihen: 28. Mai 1496. [I. F.]

251. Renerins Rowgh (Ruegh), can. eccl. colleg. S. Marie un Dauseldorp Col. dioc. - Niedere Weihen: 28. Mai; Subdiak.: 2. Juni; Diak.: 5. Juni; Presbit.: 11. Juni 1496. [I, F.] 
25̃2. Joh annes Roppgi i (Roppigiit) cler. Trev. dioc., r. capelle s. Aldegundis in Recht Col. dioc. - Subdiak.: 19. Juni; Diak.: 23. Juni; Presbit.: 26. Juni 1496. [L. F.]

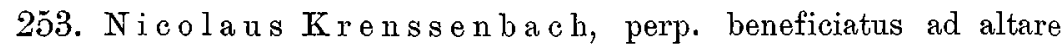
s. Nicolai in p. eccl. in Adenau Col. dioc. - Subdiak.: 25. Aug.; Diak.: 28. Aug.; Presbit.: 29. Ang. 1496 [L. F.]

254. Gerardus Ni colai de Creveldia scol. Col. - Tonsur: 22. Sept. 1496.

255. Gottfridus Buntgens cler. Col. dioc., r. perp. vicarie ad altare s. Michaelis archangeli in p. eccl. in Foresto. Niedere Weihen und Subdiak.: 18. Okt.; Diak.: 23. Okt.; Presbit.: 28. Okt. 1496. [L. F.]

2566. Joh a n nes Mi cha elis plebanus p. eccl. s. Stephani in villa Ztanstiwan (?) Col. dioc. - Tonsur, niedere Weihen u. Subdiak.: 6. Jan.; Diak.: 8. Jan.; Presbit.: 15. Jan. 1497. [L. F.]

257. Johannes Frische, r. p. ecel. in Hogenkensen (?) Col. dioc. - Subdiak.: 15. Jan.; Diak.: 17 Jan.; Presbit.: 20. Jan. 1497. [L. F.]

258. Henricus Esken cler. Col. - Niedere Weihen: 20. Mai 1497.

259. Henricus Me yer scol. Col. dioc. - Tonsur: 23. Dez. 1497. 260. Rud olphus A irch Col. dioc. -- Subdiak.: 21. Sept. 1499. 261. P a u 1 us Herman $\mathrm{n}$ us scol. Col, dioc., can. eccl. s. Balbine virg. in Suchtelen Col. dioc. - Tonsur, niedere Weihen u. Subdiak.: 11. Juli; Diak.: 13. Juli; Presbit.: 18. Juli 1501. [L. F.]

262. Joh annes Bensberch perp. cap. capellanie ville Sassindorff Col. dioc. - Niedere Weihen u. Subdiak.: 23. Januar; Diak.: 25. Januar; Presbit.: 30. Januar 1502. [L. F.]

263. Hartzabaldus $\mathrm{H}$ arde perp. vic. ad altare s. Leonardi in p. eccl. s. Pauli in Colonia, cler. Col. dioc. - Subdiak.: 13. Febr.; Diak.: 19. Febr.; Presbit.: 20. Febr. 1502. [L. F.]

264. Jordanus Gerardi de Battnboreh (!) r. p. eccl. in Harwen Traiect. dioc, cler. Col. dioc. - Niedere Weihen u. Subdiak.: 17. April; Diak.: 18. April; Presbit.: 19. April 1502. [L. F.]

265. Martinus Veuchem, vic. vicarie ad altare s. Catherine Derhellen Col. dioc. - Niedere Weihen u. Subdiak.: 9. Juli; Diak.: 10. Juli, Presbit.: 11. Juli 1502. [L. F.] 
Priesterweihen Kölner Kleriker an der Kurie im 15. u. 16. Jahrh. 111

266. Jacobus Neven cler. Col. dioc., vic. ad altare s. Anne in par. eccl. s. Vilbrordi in Assum Col. dioc. - Niedere Weihen u. Subdiak.: 10. Nov.; Diak.: 11. Nov.; Presbit.: 12. Nov. 1516. [L. F.]

267. Johannes Salmacher scol. Col., perp. beneficiatus ad altare trium missarum in p. eccl. s. Dionysii in Villanova dicte dioc. - Tonsur, niedere Weihen u. Subdiak.: 10. Mai; Diak.: 11. Mai ; Presbit. : 12. Mai 1517. [L. F.]

268. Guillelmus Rode cler. Col. dioc., perp. cap. ad altare b. Marie virg. in monasterio s. Cornelii. - Niedere Weihen u. Subdiak.: 11. Mai; Diak.: 12. Mai; Presbit.: 16. Mai 1517. [L. F.]

269. Petrus Me edardello (?) acol. Col. dioc., perp. beneficiatus ad altare b. Marie virg. in p. eccl. Mondersheim dicte dioc. - Subdiak. : 3. Juni ; Diak.: 4. Juni ; Presbit.: 5. Juni 1517. [L. F.]

270. Willelmus Spykernagell cler. Col. dioc., perp. cap. ad altare b. Marie virg. in p. eccl. s. Laurentii in villa Mintert eiusd. dioc. - Niedere Weihen u. Subdiak.: 20. Sept.; Diak.: 21. Sept.; Presbit.: 24. Sept. 1517. [L. F. mit Zusatz: der Geweihte diurfe erst nach 5 Monaten und nur mit Erlaubniss seines Ordinarius celebriren.]

271. Jacobus Pluys de Schweynheym acol. Col. dioc., perp. vic. ad altare s. Caterine virg. in p. eccl. s. Cosme et Damiani in villa Holtzwiller. - Subdiak.: 17. April; Diak.: 19. April; Presbit.: 22. April 1518. [L. F.]

272. Johannes de Tzons cler. Col. dioc., perp. vic. ad altare s. Crucis et b. Marie virg. in p. eccl. s. Laurentii civitatis Coloniensis. - Niedere Weihen: 30 April; Subdiak.: 10. Mai; Diak.: 11. Mai ; Presbit.: 12. Mai 1518. [L. F. 30. April 1518!]

273. Gotfridus de Oisterwick can. prebende eccl. ss. Cassii et Florentii oppidi Bonnensis Col. dioc. - Diak. : 29. Mai 1519. [L. F.]

274. Victor de Cerpena ac. Col. dioc., perp. vic. ad altare b. Barbare virg. in p. ecel. s. Laurentii civitatis Coloniensis. Subdiak.: 28. Juni; Diak.: 29. Juni; Presbit.: 2. Juli 1519. [I. F.]

27ö. Johannes Bel, cler. Col. dioc., perp. vic. ad altare s. Nicolai in capella s. Sebastiani extra muros oppidi Wachtendunck. - 
Niedere Weihen u. Subdiak. : 7. Aug.; Diak. : 14. Aug.; Presbit.: 4. Sept. 10̃19. [L. F.]

276. Renerius Saffenberg de Bonna cler. Col. dioc., perp. cap. ad altare $x$ milium martirum in p. eccl. s. Nicolai in Statinen Caminensis dioc. - Subdiak.: 1. Màrz; Diak.: 2. März; Presbit.: 15. Mä̀z 1520. [L. F.]

277. Wolfgangus Polich, cler. Col. dioc., r. p. eccl. in Syburch. - Niedere Weihen tr. Subdiak.: 22. Mai; Diak.: 23. Mai 1520. [L. F.]

278. Petrus Tute plebanus sive pastor nuncupatus perpetue vicarie curate ad altare s. Crucis in eccl. s. Victolis Xanctensis Col. dioc. - Niedere Weihen u. Subdiak.: 5. Jan.; Diak.: 6. Jan.; Presbit.: 7. Jan. 1521. [L. F.]

279. Joh andes Gerthusen cler. Col. - Niedere Weihen u. Subdiak.: 15. Juni; Diak.: 16. Juni; Presbit.: 17. Juni 1521. [L. F.]

280. Le onardus Fabri acol. Col. dioc., perp. cap. perp. capellanie ecclesie paroch. s. Pancratii in villa de Jeren (ob zu lesen Glehn?) ad altare s. Anne dicte dioc. - Subdiak. : 8. Sept.; Diak.: 9. Sept.; Presbit.: 12. Sept. 1521. [L. F.]

281. Joh an nes Bocholt cler. Leodiensis dioc., perp. capell. capelle s. Georgii iuxta castrum de Nienhaven Col. dioc. Niedere Weihen п. Subdiak.: 15. Nov.; Diak.: 16. Nov.; Presbit. : 17. Nov. 1521 [L. F.]

282. Adam Surgen cles. Col. dioc., perp. capell. ad altare s. Catharine in p. eccl. s. Johamnis loci de Surgen dicte dioc. Niedere Weihen und Subdiak.: 27. Juni; Diak.; 30. Juni; Presbit.: 15̄. Juli 1522. [L. F.]

283. $\mathrm{Nicolaus} \mathrm{Her}$ manni perp. vic. ad altare ss. Antonii et Lamentii in capella s. Johamnis in Horstgen. - subdiak.: 17. Okt.; Diak.: 18. Okt.; Presbit.: 1. Nov. 1522. [L. F.]

284. Hexmannus op den Graeff de aintiqua ecclesia cap. capelle s. Antonii sub et infra limites parochie eccl. s. Petri in Oldenkerken Col. dinc. - ebenso.

285. Arnoldus Tenhenhaeft cler. Col. lioc., s. Clementis Wysselensis et b. Marie virg. Ressensis dicte Col. dioc. ecclesiarum collegiatarum can. - Niedere Weihen n. Snbdiak.: 1. Nov.; Diak.: 2. Nov.; Presbit.: 4. Nov. 1522. [1. F.] 
riesterweihen Kölner Kleriker an der Kurie im 15. u. 16. Jahrh. 113

286. W ilh el $\mathrm{m}$ us $\mathrm{Mu}$ ch e cler. Col. dioc., cap. ad altare s. Catharine in p. eccl. de Muche Col. dioc. - Diak.: 31. März; Presbit.: 1. April 1523. [L. F.]

287. Arnoldus Moler cler. Col. dioc., perp. vic. eccl. s. Catherine Hamburgensis Bremensis dioc. - Niedere Weihen und Subdiak.: 31. Januar; Diak.: 1. Febr.; Presbit.: 2. Febr. 1524. [L. E.]

\section{Verzeichniss der erwähnten Kirchen, Klöster, Kapellen u. s. w.}

(Die beigefügten Nummern beziehen sich auf die Nummern der Regesten.)

Adenin 253.

Gereonsweiler 131.

Aldekerk (Kr. Geldern, jetzt Diöz.

Gerresheim 110.

Münster) 284.

Alpen (jetzt Diöz. Münster) 227.

Alsdorf 239 .

Altenahr 220.

Andernach 221 .

Asbach 248.

Bienen (Kr. Rees, jetzt Diözese II ïnster) 40.

Bonn, St. Cassiusstift 84 (?), 184, 225, 273.

- Stitt Dietkirchen 181.

Brakel (Kr. Dortmund, jetzt Diöz. Paderborn) oder Brachelen bei Juilich 1.

Breisig (Kr. Ahrweiler) 142.

Bremen 115.

Cornelimünster 100, 182, 268.

Dattenfeld 147.

Derhellen, siehe Höllen.

Dorenspyck (Diöz. Utrecht) 36 .

Disseldorf 168, 251 .

Elbing (Diöz. Ermland) 169.

Fisberg (?) 194.

Glehn (bei Neuss) 280.

Glenel 33.

Götterswickerhamm, (Krr. Mülhein a. d. R.) 196.

Graenky ndorp (?) siehe Selgersdorf.

Hachenburg 204 .

Haldern (Kr. Rees) 212.

Hamburg 287.

Haren 190.

Hassum (Kr. Cleve, jetzt Diözese Münster) 266.

Herwen (bei Zevenaar, Diöz. Utrecht) 264.

Hilfahrt 249.

Hog'enkensen (?) (ob Höngen bei Aachen?) 257.

Holzweiler 271.

Höllen (bei Rödinger, Ǩr. Jülich) 265.

Hörstgen 283.

Hubbelrath 199.

Hugert ( $o b$ Hergarten bei Zülpich?) 195.

Hursen 156.

Immerath 245 .

Jülich 148 .

Frechen 77.

Kaiserswerth 66 . 
114 Ludwig Schmitz: Priesterweihen Kölner Kleriker a. d. Kurie etc.

Kevenberg 33, 203.

Köln, St. Andreas 2, 11, 123, 152.

- St. Aposteln 17ă.

- St. Brigida 224.

- St. Christoph 155.

- St. Cunibert 76, 188, 192, 207 (?).

- Doin 69, 151, 201, 217.

- St. Gereon 39.

- St. Johann 187.

- St. Laurentii 272, 274.

- St. Lisolphi 210.

- St. Maria im Kapitol 33, 200.

- St. Martin minor 235.

- St. Mauritius 189.

- St. Michael 236, 237.

- St. Paul 263.

- St. Peter u. Paul (?) 202.

- St. Reinoldskapelle 63.

- St. Severin 163.

Korschenbroich 185.

Liblar 193.

Lobberich 191.

Lübeck 115.

Mechelen (bei Antwerpen) 20.

Meiderich (Kr. Ruhrort, jetzt Diöz. Münster) 3.

Menden (Kr. Iserlohn, jetzt Diöz. Paderborn) 2.

Minbach (ob Mintard?) 219.

Mintard 270.

Molenarck 214.

Monheim 158.

Much 70, 286.

Müddersheim (bei Düren) 269.

Münstereifel 90, 176, 198.

Münstermaifeld (Diöz. Trier) 6, 131 .

Neuenhoven (bei Neuss) 281.

Neundorf bei St. Vith 137.

Neuteich (Kr. Marienburg) 169.

Niederpleis 117.

Nieukerk (Kr. Geldern, jetzt Diöz. Münster)? 218, 267.

Oberbachem 154 .

Oberwinter 106.

Phanert (?) 215.

Radevormwald 209.

Recklinghausen 222.

Recht 252.

Rees 38, 95, 285.

Rheydt 162.

Rindern (Kr. Cleve) 111.

Rodenkirchen 64.

Sassendorf 263.

Selgersdorf (?) 132.

Siegburg 277.

Soest 161.

Steinbüchel 211.

Stettin 276.

Stildorf 127.

Stommeln 78 .

St. Vith 137.

Süchteln, Pfarre 7.

- Stift Rade 261.

Sülen (untergegangener Ort in Kr.

Rees, jetzt Pfarre Praest) 27.

Surgen? 282.

Udenbreth 246 .

Ulperich 205.

Viersen 34, 54, 98, 159.

Wachtendonk Kloster Thal Josaphat 126.

- St. Sebastianskapelle 275.

Wamel (jetzt Niederlande) 141.

Wankum 247.

Wesel 208.

Wetten (Kr. Geldern) 146.

Wissel (Kr. Cleve) 285.

Wollersheim 164.

Xanten 11, 35, 68, 72, 73, 75, 278.

Zülpich 216. 